\title{
Computational biology for enzymatic removal of mycotoxins
}

\author{
Natalie Sandlin*, Darius Russell Kish*, John Kim, Marco Zaccaria, and Babak Momeni ${ }^{\dagger}$ \\ Department of Biology, Boston College, Chestnut Hill, MA 02467 US \\ * equal contribution \\ $\dagger$ corresponding author, momeni@bc.edu
}

\begin{abstract}
Biological organisms carry a rich potential for removing toxins from our environment, but the search to identify suitable candidates remains challenging. We survey and explore the use of computational tools to discover and optimize the detoxification of harmful compounds. In particular, we will focus on mycotoxins - fungi-produced toxins that contaminate food and feed - and biological enzymes that are capable of rendering them less harmful. We discuss the use of computational tools to complement existing empirical data in three main directions: discovering the prospect of detoxification among underexplored organisms, finding important cellular processes that contribute to detoxification, and optimizing the performance of enzymes with detoxification capability.
\end{abstract}

\section{Background and Motivation}

\subsection{Context: detoxifying contaminated food and feed}

Food that we consume and feed that supports livestock are at risk of getting contaminated with harmful compounds. Fungi that grow on foodstuffs are one of the major sources of toxin contamination in food and feed; these fungi-produced toxins are called mycotoxins. Currently, an estimated $25 \%$ of world crops is thought to get contaminated with mycotoxins each year $(1,2)$, putting a major burden on agriculture and public health. Preventing contamination or detoxifying mycotoxins is a major safety priority (3). In what follows, we briefly describe the threat of mycotoxins (Section 1.2), the potentials of biological organisms to address this threat (Section 1.3), and an outline of different detoxification mechanisms, with a focus on enzymatic detoxification (Sections 1.4 and 1.5). We will then investigate and explore the use of computational approaches to discover and improve such potentials. We will first discuss the use of bioinformatics tools to mine the genomic databases for candidate species and enzymes (Section 2.1). We discuss how efficient detoxification may rely on more than one organism (Section 2.2). Once such species and strains are identified, either computationally or experimentally, genetics and genomics data can be used to investigate how their performance can be improved (Section 2.3). The enzymes responsible for detoxification can also be computationally optimized (Sections 2.4). Finally, we will explore future advances in 
computational tools (Section 3.1) and machine learning (Section 3.2) that can propel this field forward.

\subsection{Mycotoxins are prevalent and harmful}

Mycotoxins are secondary metabolites produced by a variety of filamentous fungi that contaminate common food crops and cause negative health effects in animals and humans. More than 300 types of mycotoxins have been identified so far (1). Among these, six major types are of particular interest, because of their detrimental health impact and because they routinely contaminate foods and animal feed $(4,5)$ : aflatoxin (AF), ochratoxin (OT), zearalenone (ZEA), fumonisin, deoxynivalenol (DON), and patulin.

Aflatoxins, produced by Aspergillus species, are one of the most carcinogenic naturally occurring substances and active inducers of mutations, liver cancer, congenital malformations, hormone disorders, and immunodepression $(6,7)$. Ochratoxin is also produced by Aspergillus species, as well as certain Penicillium species, and is a nephrotoxin, immunosuppressant, potent teratogen, and renal carcinogen $(6,8,9)$. Zearalenone and fumonisins are produced by Fusarium species. ZEA acts through estrogen mimicry to dysregulate the hormone receptor and antagonize the estrogen pathway leading to reproductive disorders, hormone imbalance, and breast cancer $(6,10)$. Fumonisins have been linked to esophageal cancer in humans as well as a variety of health complications in animals such as pulmonary edema and hepatotoxicity $(6,11)$. Produced by Fusarium graminearum, DON is a vomitoxin, causing emetic and nauseous effects after ingestion (12). Finally, patulin is produced by ascomycetes such as Penicillium, Aspergillus, and Byssochlamys species and is commonly found in fruit and vegetable products, especially rotten apples and apple juice (13). Patulin ingestion is linked to a number of health complications, namely immune suppression, ulcers, gastrointestinal inflammation and embryotoxicity (13). There are a variety of food crops that these mycotoxins contaminate, including cereal crops such as wheat, barley, corn, and oats $(6,11)$. The negative health effects caused by mycotoxin consumption have warranted regulations on permissible amounts to be found in foods that differ by country and toxin.

Due to the serious health implications of mycotoxin contamination, economic losses arise from reduction of crop and livestock yields as well as the cost of decontamination efforts. Annually, the United States faces economic losses of about $\$ 932$ million, from AFs, fumonisins, and DON alone (14). However, this number is merely an estimate because the definition of a mycotoxin loss varies according to the buyer and seller. In any case, this sizable economic burden is faced across agriculture and livestock producers globally and requires efficient and cost-effective measures as a solution.

Within the food production process, there are multiple sites where contamination can occur and mycotoxins can build up on food products. The fungi that produce mycotoxins are found ubiquitously in the environment and cause contamination not only in the field while crops are growing, but mostly post-harvest while in processing and storage. Mycotoxins build up on foodstuff necessitates methods of decontamination in order to supply safe foods for consumption. Currently, decontamination is limited to physical and chemical methods. Physical 
methods, including sorting and cleaning, have been shown to be effective in some but not all cases of mycotoxin contaminations. These methods of removal have the negative attributes of decreased crop yield, high operational costs, and limited reliability of delivering products safe for consumption $(3,14,15)$. Chemical methods, which use chemical agents to reduce or convert mycotoxins into less toxic byproducts, include ozonation and ammoniation. While these methods have been used successfully by several countries for the reduction of mycotoxin contamination, they possess similar limitations to physical methods, high operational costs and limited reliability, and the additional limitation of decreasing the quality or nutritional value of the food $(3,14,16)$. These limitations decrease efficacy and efficiency of mycotoxin decontamination and expose the need to look for better solutions.

\subsection{Toxin removal by biological processes is a promising solution}

Bioremediation, or the use of biological entities to detoxify or remove toxins in the environment, is a promising alternative to current decontamination methods. Bioremediation offers lower costs, fewer undesired environmental side-effects, and potentially higher efficiency and reliability $(17,18)$. The use of microbes is a particularly attractive choice in bioremediation, offering faster activity and the feasibility of strain evolution and engineering for improved performance (19). There are six key factors that make a good bioremediator: 1) fast and efficient at degradation, 2) safe degradation products, 3) non-pathogenic to plants, animals, or humans, 4) not detrimental to the quality of the food/feed, 5) applicable outside of lab settings, and 6) applicable to the broadest range of pollutants (17). Many identified mycotoxin degraders fit one or more of these parameters, however none effectively fit them all, with speed and efficiency often being the limiting factor. Additionally, the mechanisms of degradation by these identified microorganisms are often unknown or understudied, limiting the ability to improve upon the native degradation performance.

The biological control of mycotoxin contamination has so far been based on two major strategies: prevention of toxin production and toxin removal/detoxification after contamination $(20,21)$. For example, numerous studies have examined the ability of lactic acid bacteria (LAB) against mycotoxins on both fronts (22). LAB strains have been shown to suppress the growth and toxicogenesis of mycotoxin-producing fungi (22-25), while also removing the toxin from contaminated food and feed by either adsorption of toxins to cells $(20,24,26)$ or production of toxin-degrading enzymes (24).

\subsection{Modes of biological detoxification}

In the context of microbial interventions for mitigation of mycotoxins, there are two main modes of detoxification: adsorption and biotransformation. In adsorption, mycotoxins are physically bound to the outer structures - polysaccharides and proteins - of the microbes to reduce bioavailability of the toxins upon consumption $(20,24,26)$. Biotransformation utilizes microbes and their enzymes to degrade mycotoxins into non-toxic metabolites $(27,29)$. This 
mode can be further broken into three categories by secretion of enzymes (extracellular degradation), binding of toxin to the cell surface (membrane-bound degradation), and uptake of the toxin into the cell (intracellular degradation). Microbes that mitigate mycotoxins through extracellular degradation are more likely to produce enzymes that are stable outside of the cell body and have the potential for isolated enzymes to be used as bioremediators. Intracellular degradation of toxins into the cell body more closely follows normal metabolic processing of molecules by microbes and could inhibit efficiency through regulation of toxin uptake.

Microbial adsorption of AFs, namely $\mathrm{AFB}_{1}$, has been shown to occur through a stable, yet reversible mechanism in Lactobacillus strains (30,31). Yeast cell wall from Saccharomyces cerevisiae has also shown wide adsorption affinity for a variety of mycotoxins $(31,32)$. Kabak et al. report reversible adsorption of mycotoxins to Lactobacillus and Bifidobacterium (33). Denaturation of surface proteins by heat in Lactobacillus results in increased adsorptive property, suggesting mycotoxins bind to protein moieties or polysaccharides whose surface areas are increased by denaturation $(31,34)$. Additionally, heat-treated Aspergillus conidia display adsorptive properties (35).

Enzymatic degradation has been suggested in a number of studies; however, identification of the degrading enzymes has proven difficult. Sangare et al. show a Pseudomonas species capable of degrading $\mathrm{AFB}_{1}$ from cell-free culture supernatant, suggesting that an extracellular enzyme is responsible for the degradation (36). Screening for the effect of common

cofactors such as $\mathrm{Mn}^{2+}, \mathrm{Cu}^{2+}$, and $\mathrm{Zn}^{+}$may potentially help identify the enzyme class. Similar extracellular degradation has been reported for Rhodococcus spp., Stenotrophomonas spp., and Myxococcus spp. (37-39). Similar to AF, DON has been shown to be enzymatically degraded by an unidentified extracellular enzyme (40); DON has been observed to be assimilated as a carbon source in some, but not all strains (41). Other extracellular enzymes with mycotoxin degrading abilities include oxidoreductase, dehydrogenase, aldo-keto reductases, and peroxidases (40, 42, 43). While there has been less focus on intracellular mechanisms, intracellular enzymatic degradation has been shown by Zhu et al. (44). In the remainder of this work, we will limit the scope to the capabilities of bacterial and fungal enzymes to convert mycotoxins to less harmful compounds. We make this choice to offer a more focused view on recent developments in computational tools for biological enzymes, but also to keep practical considerations and regulations in mind for deploying enzymes versus live organisms in food/feed applications (27, 28).

\subsection{Bacteria and fungi carry a rich repertoire of enzymes capable of removing mycotoxins}

Biotransformation of mycotoxins into non-toxic products has already been demonstrated by using the rich repertoire of enzymes in bacteria and fungi $(28,42,45)$. For example, laccase from the basidiomycete Trametes versicolor degrades $\mathrm{AFB}_{1}$ by more than $80 \%$ after 72 hours of incubation $(29,40)$. Thamnidium elegans and Mucor bainieri are other fungi that have displayed 
degradation ability by transforming ZEA into a non-estrogenic substance ZEA-4-O- $\beta$-glucoside (40).

Enzymes produced by microorganisms have promising potential for future applications in the food industry. Many enzymes have been shown to have the capability to remove mycotoxins. Nevertheless, biological detoxification can be further improved by exploring the identification and characterization of degrading/detoxifying enzymes. A better understanding of the mechanisms of enzymatic detoxification can lead to more efficient implementations that address the current needs. On one side, the cellular machinery of the degradation (schematically shown in Fig 1) can be optimized to improve the overall performance. On the other hand, the enzyme itself can be modified for improved performance. Structural modelling and design of experiments (DOE) technologies can shed light on the identification of key structural components that contribute to degradation ${ }^{24}$. However, more research is still needed to optimize degradation efficiency with emphasis on degrading enzymes. In the following section, we will address some of the existing computational tools that can facilitate the discovery and use of biological organisms for enzymatic removal of mycotoxins.
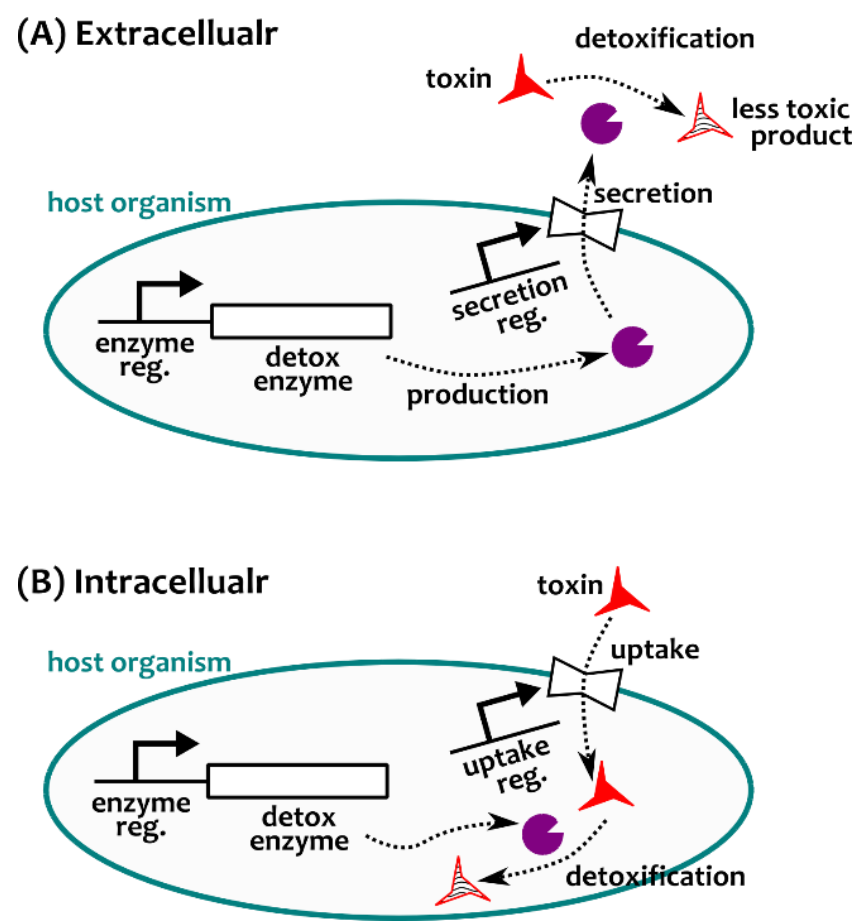

Figure 1. Simplified representation of the cellular machinery involved in (A) extracellular versus (B) intracellular detoxification. 


\section{Current Challenges and Computational Solutions}

How can we effectively remove mycotoxins using biological organisms? Conceptually, we break down this search into two steps: (1) finding organisms that have this capability, and (2) optimizing the performance by modifying the environmental conditions, the detoxifying strain, or the target enzymes. We survey existing computational tools that can facilitate this process (Fig 2).

\section{Enzymatic removal of toxins: Questions addressed by computational tools}

\section{What organisms have the capability? \\ Searching for strains that carry detoxifying enzymes \\ Searching for strains with complementary functions}

How can we optimize the performance?

Investigating the cellular machinery of the strains

Investigating the molecular structure of the enzymes

Figure 2. Conceptual breakdown of major questions of interest where computational tools can facilitate more efficient removal of toxins.

\subsection{Finding candidate organisms: who can do the job?}

Discovering organisms that can degrade mycotoxins poses a number of challenges that can be met both through experimental and computational approaches. Although this section focuses primarily on enzymatic degradation rather than adsorption, similar approaches may be used for finding and optimizing adsorption-based removal of mycotoxins. In terms of enzymatic degradation, there are three challenges to be addressed. First, organisms must have the genes necessary to produce enzymes and possibly cofactors involved in degradation. Second, the organisms must have favorable regulatory mechanisms for these enzymes. Third, the method of obtaining and isolating the enzymes must be favorable to the end use case. One can describe the search space as being largely defined by these characteristics that may be specific to the use cases, but are still conceptually similar among different cases.

As an example, there is a known, highly specific two-step enzymatic process in the detoxification of fumonisin, which involves a carboxylesterase and an aminotransferase (46). This becomes a useful bottleneck in the search space, as candidate organisms must contain both enzyme-encoding genes to be viable degraders. Toward this end, tools such as BLASTp (47) can 
be utilized in cases where genome sequences are available. Simply put, the presence of these two genes largely dictates whether or not an organism is a fumonisin degrader. On the other hand, in the example of AF detoxification, many species can possess hydrolases or oxidases related to those that are known to degrade $\operatorname{AF}(29,42,48)$. The search space is instead constrained on a separate manifold involving the specificity and affinity of the hydrolase for AFs. That is, the presence of the same hydrolase gene may not be sufficient to identify degradation potential, since it may be optimized for a different substrate. The relationship then lies on a manifold relating sequence to function, which is not guaranteed to be captured by sequence similarity $a ̀$ BLASTp. This shortcoming can be thought of as a signal to noise ratio, where key amino acids involved in the active site mechanism are sparse signals, and the rest of the sequence functions primarily to provide the correct structural shape and may be noisy in this regard. This is witnessed in the work by Dellafiora and colleagues (49), where two related, AF-degrading hydrolases shared only $72 \%$ sequence similarity, despite using the same mechanism for degradation. In a more extreme example, a recently identified carboxylesterase that degrades fumonisin shows only around $34 \%$ sequence similarity to previously reported fumonisindegrading carboxylesterases (50).

The shortcoming of sequence similarity-based methods is that they may be brittle when used to identify enzymes by function, rather than by phylogenetic relations. Sequence similarity may be used to imply functional similarity; however, such a predicate does not include enzymes that share functional similarity without sequence similarity. In a similar fashion, high sequence similarity among closely related species might not fully overlap with functional similarity. Therefore, searches should be conducted on a sequence-to-function relationship model. While this method loses the high-throughput optimizations of BLAST-based sequence similarity, it may be modeled via a reductive filter pipeline to maintain reasonable complexity. It also loses the generalizability of sequence similarity, and instead pipelines must be custom designed for each end use case. In the example of AFs, initial work has been performed to design a structure to function reductive filter model using a number of inductive prior filters. Furthermore, this model does not necessarily require a labeled, positive enzyme to seed the search, rather it only requires characteristics to build the filters. Prior research by Risa and colleagues (51) has identified that excreted enzymes can be responsible for degrading AFs. SignalP is able to predict protein excretion in bacteria, and can be used as an initial filter to narrow down proteomes. These sequences can be passed through both size and sequence-based enzyme classification filters based on facile experimental determinations to further reduce the candidate pool. From here, 3D structures may be built, the binding pockets predicted, and AF docked to identify high affinity interactions that then may be confirmed experimentally. These computational processes will be expanded upon in section 3.The reductive filter model uses low-complexity tools at its head, increasing in complexity towards the tail to ensure efficiency. Similarly, its modular nature allows for easy insertion or upgrading of components as advances occur in each domain. 
From the experimental front, high-throughput screening may be used both to identify candidate organisms as well as explore mutations for optimizing degradation potential. Environmental isolates are a traditional source for identifying mycotoxin degraders. Isolates can be cultivated and tested for degradation, especially when high-throughput screening is possible. For example, AF was shown to be degraded by Rhodococcus erythropolis via an excreted enzyme, and additionally AF presence can be detected via the fluorescence of its lactone ring, which is broken during degradation (52). Such a system can be easily assayed by fluorescent assays of the supernatant. Other systems, however, may require lysing, filtration and HPLC or Mass Spectroscopy methods to perform such assays, limiting their throughput. A similar screen may be used both for optimizing the environmental conditions or the enzyme itself.

\subsection{Community-level detoxification: when the task needs to be divided}

Mycotoxin degradation may require multiple reactions to reach byproducts with complete or significantly decreased toxicity. In these cases, two or more enzymatic steps comprise the detoxification process and necessitates the understanding of how multiple enzymes from the same, or even different, species are required for degradation of a single mycotoxin. In the degradation of fumonisin $\mathrm{B}_{1}$ by Sphingopyxis sp. MTA144, Heinl et al. identified two enzymes as components of this process (46). The first gene encodes a carboxylesterase that facilitates the initial deesterification step to form a hydrolyzed fumonisin $\mathrm{B}_{1}$, which is less active in its known ceramide synthase inhibitory pathway but still possesses significant toxic effect $(46,53)$. Following this step, the second enzyme, an aminotransferase, deaminates the hydrolyzed byproduct of the first reaction resulting in complete degradation and loss of toxic effects (46). Similarly, Carere et al. elucidated a two component enzymatic pathway involved in the epimerization of DON by Devosia mutans 17-2-E-8 $(54,55)$. The enzymes, designated DepA and DepB, first oxidize DON into 3-keto-DON (DepA) (54) and subsequently reduce 3-ketoDON into 3-epi-DON (DepB) (55), significantly reducing toxicity. These examples highlight the potential of microorganisms to degrade mycotoxins in a multi-step process and the need to understand all the enzymes playing a role in complete degradation.

There are many instances of mycotoxin degradation by microorganisms that do not lead to complete detoxification (28). As in the above example of multi-step DON degradation, the end products, while less toxic than the starting substrate, retain some level of toxicity and can still be harmful. Additionally, in the degradation of ZEA, there are cases where microbial breakdown results in byproducts, $\alpha$-zearalenol and $\beta$-zearalenol, that are more toxic than the original compound (56-58). In these cases, it would be necessary to identify additional species or enzymes that can take the byproducts and convert them into non-toxic compounds, forming a multi-step process toward detoxification through a microbial consortium. Wang et al. discovered a microbial consortium toward the detoxification of ZEA and its toxic analogs that utilizes multiple species across various taxa working in unison to transform ZEA to non-toxic byproducts (59). Multi-step degradation to non-toxic compounds emphasizes the need to look 
beyond single microorganisms and research into the creation of microbial consortia to complete the job.

Bioinformatic searches for identifying multiple enzymes necessary for a particular case would be an extension of the single-enzyme searches discussed in the previous section, using the similar tools. Of note could be searching for individual organisms that carry two or more necessary enzymes that have previously been identified in multiple species/strains.

\subsection{Regulation: even when the detoxification capability exists in an organism, its availability may be under regulation of production and/or secretion}

Even after organisms have been identified that are capable of detoxifying target pollutants, the availability of the relevant enzymes depends on whether the environmental context induces the relevant genes of enzyme production and secretion effectively. These considerations point to the need to explore the internal regulation of the production and secretion of detoxification enzymes. Microorganisms respond to cellular and environmental changes through regulatory decisions that could impact the availability of degradation machinery for target pollutants (60). Production of enzymes is regulated through different mechanisms, such as transcription factors binding in and around promoter regions that contributes to the amount of enzyme produced by the cell. These mechanisms are likely influenced by nutrient availability and overall conditions of the cell (i.e. growth phase) (61). Secreted enzymes have an added layer of regulation due to the high energy cost of secretion. While these enzymes have beneficial effects, often being employed to breakdown macromolecules in the environment for cell nutrients, they also impose cost on energy and biomass (62). Therefore, certain enzymes targeted for secretion are up- or down-regulated by the presence of nutrients in the environment that respectively do or do not require extracellular breakdown. Understanding the influence of these gene regulatory factors on production and secretion of the enzyme is necessary for strain optimization and for factoring in the cost-benefit balance for increased enzyme production and secretion.

Several existing bioinformatic tools can help us uncover aspects of bacterial gene regulation, such as promoter and DNA binding sites, operon regions, and secretion signals, which are touched on in the following sections. The usefulness of these tools in the context of bioremediation is that they allow researchers to uncover possible mechanisms of regulation that control the detoxification process. Insight from regulation, for example similarity to a known catabolic pathway, can also be used to choose suitable environmental conditions or infer the mechanism of degradation.

Promoter Prediction. Identifying promoter regions and DNA binding sites are important in that transcription initiation is the most frequently regulated step in gene expression. Promoters contain an intrinsic strength that governs the amount of transcription a gene undergoes and when transcription occurs according to environmental factors such as nutrient availability (61). By 
uncovering promoters associated with genes/enzymes of interest in bioremediation, we can understand how the cell naturally regulates its expression and better manipulate it toward improved expression. While there are many tools for the prediction of and databases of promoter regions in different organisms, such as phiSITE $(63,64)$, SAPPHIRE (65), PRODORIC2 (66), BacPP (67), and PPCNN (68), we will expand on the latter three.

PRODORIC2 is a transcription factor binding site (TFBS) database that possesses one of the largest collections of DNA binding sites in prokaryotic organisms (66). In 2018, its most recent update, PRODORIC2 expanded its database to host the genomic information of 2274 bacterial strains and their 5191 replicons (66). This database is curated to only include experimentally validated binding sites, limiting the expanse of bacterial species it contains but ensuring accuracy in its TFBS inventory. De Avila e Silva et al. created a bioinformatic tool, BacPP, to predict promoter sequences in Escherichia coli strains through neural network simulations (67). BacPP is able to recognize and predict promoter sites with varying levels of accuracy (all above 83\%) across the different sigma factors crucial for prokaryotic transcription initiation (67). Additionally, BacPP has $76 \%$ prediction accuracy among other enterobacteria species (67). The advantage of this method is in its ability to classify promoter sequences by its sigma factor, an important distinguishing feature that was a shortcoming of previous tools. However, BacPP is currently limited to E. coli and enterobacteria (with lessened accuracy). Another promoter prediction tool is Promoter Prediction Convolutional Neural Network (PPCNN), developed for both eukaryotic and prokaryotic prediction and implemented into the CNNProm program. This approach similarly uses deep learning neural networks to establish the prediction models (68). For prokaryotes, PPCNN was trained on E. coli and Bacillus subtilis, offering insight into both Gram-positive and Gram-negative species. A highlight of this method is its functionality to other sequenced species due to its ability to predict promoters without prior knowledge of specific promoter features (68).

Operon Prediction. Metabolically or functionally related genes within prokaryotic genomes are often arranged in contiguous segments called operons and are co-transcribed along the same messenger RNA (69). This organization imparts an added layer of regulation on the genes within the operon and necessitates understanding of how target genes and proteins are under this regulation. Specifically, in the context of bioremediation, if an enzyme of interest is encoded within an operon, it opens up new genes that could help play a role in degradation, either functionally or through regulation. Determining operons computationally has been a field of interest for a number of years, leading to tools such as Operon DataBase $(70,71)$, OperomeDB (72, 73), Operon Hunter (74), and Operon-mapper $(75,76)$, with recent advances in de novo prediction of operons from genomic data which is expanded on below.

Operon-mapper, a web-based server for operon prediction, was developed in 2018 and is the first publicly available tool for operon prediction that only requires genome sequences as the input $(75,76)$. Operon-mapper uses a five step procedure: (1) open reading frame (ORF) prediction using Prokka software (77, 78); (2) homology gene determination using the 
hmmsearch program based on Hidden Markov Models (75, 78); (3) intergenic distance evaluation using a custom program (75); (4) operon prediction using an artificial neural network with intergenic distance and a score defining functional relatedness of protein products as the input arguments $(75,79,80)$; and (5) gene function assignment using the DIAMOND algorithm (81). The accuracy of this method in predicting operons was $~ 90 \%$ across eight tested genomes with varying size and GC content, and outperformed other algorithms in a recent evaluation of correlation to experimentally validated operons (82). Operon-mapper also has the advantage of providing significant information, such as ORF identification and functional annotation of protein, that is relevant to bacterial genome annotation (75).

Secreted Protein Prediction. A signal peptide (SP) is a sequence of amino acids in a newly synthesized protein that targets the protein into or across the membranes in the cell (83). Determining whether an enzyme is secreted, and by which secretion pathway it exits the cell, enables better utilization of the degradation machinery. It helps us to better understand how the enzyme interacts with the target pollutant and whether secretion can be manipulated for enhanced degradation. To predict secreted proteins, several algorithms to identify SPs within a proteome have been developed: SignalP (84), Psort (85), Pred-Tat (86), and TatP (87).

Of note, SignalP is able to determine these secretion signals and distinguish between the type of secretion pathway. The current version, SignalP 5.0, uses deep neural networks in combination with conditional random field classification and optimized transfer learning to determine SPs in prokaryotes, eukaryotes, and archaea (84). This update builds upon previous versions based on artificial neural networks (88), with added improvements of hidden Markov models (89), enhanced cleavage site predictions (90), and discrimination of signal peptides and transmembrane helices (91). For prokaryotes, there are two main secretion pathways, Sec and Tat, with three enzymes, signal peptidases I-III (SPase I-III), needed to cleave proteins for secretion. SignalP 5.0 is able to distinguish between three types of SPs: (1) Sec substrates cleaved by SPase I; (2) Sec substrates cleaved by SPase II; and (3) Tat substrates cleaved by SPase I (84). Unfortunately, due to limited data sets for training, SignalP 5.0 is unable to predict Sec substrates processed by SPase III or Tat substrates processed by SPase II. However, the current ability to determine between the three secretion pathways is important in understanding how the protein will be secreted and the regulation of the secretion process. Prediction performance of SignalP 5.0 showed competitive performance and, in some instances, enhanced prediction precision compared to other more specialized prediction algorithms (84). SignalP 5.0 is available either through their webserver or as a standalone package, making it an accessible tool for secreted protein prediction. 


\subsection{Sub-optimal enzymes: naturally evolved enzymes may not be the best match}

Enzymes found capable of degrading mycotoxins may not be naturally optimized for targeting the mycotoxin of interest. Enzyme optimization often involves adaptation of a wildtype isolate to a new substrate or reaction environment. New reaction environments often involve changes of temperature, $\mathrm{pH}$, and solvent conditions, all of which non-trivially affect the structure and activity of the enzyme. One technique that is agnostic to fundamental understanding of these effects is directed evolution (92-94). In directed evolution, genetic diversity is introduced via random mutations and the resultant mutant proteins are screened for improved performance. There is some evidence that restricting directed evolution to residues close to the active site leads to a higher probability of displaying meaningful contributions to its activity (95). However, it remains unclear how such a process is achieved through traditionally structure-agnostic in vitro mutagenesis. Often, directed evolution is applied iteratively, taking the form of a genetic algorithm to further improve strong performing mutants (96). Though directed evolution conveniently creates a black-box optimization method, it does so at the cost of efficiency, where screening for fitness can become a major bottleneck in the process (97).

Protein sequence activity relationship (ProSAR) models may provide additional power to the search algorithm by creating a statistical model linking the protein sequence to its activity (i.e., fitness) $(98,99)$. ProSAR relies on a mutant library generated from mutagenesis with a constraint of constant protein sequence length, along with the corresponding activities of interest (catalytic constant, thermostability, etc.). A statistical model is built that links the presence or absence of individual mutations to a contribution to the activity, from which some subset of the highest contributing mutations can be fixed for the next round of mutagenesis. Unlike the close mutations described earlier by Morely et al. (95), this method is able to link individual mutations to activity contributions without explicit knowledge of the 3D structure. The traditional statistical methods for ProSAR involved partial least square regression and genetic algorithm, while more recently traditional statistical methods could be replaced with Recurrent Neural Network architectures (100).

Focused evolution, where targeted mutations are introduced based on rational mutation hypotheses, can increase the efficiency of optimization by narrowing the search space; however, current robust methods require 3D structures of the enzyme. When optimizing for known properties such as thermostability and where reasonable 3D models are available, such as homology models, a small subset of rational mutations can feasibly be explored through computational methods and the final mutations evaluated experimentally. Rational mutation methods rely on heuristic evaluation methods like FoldX (101) to predict changes in Gibbs free energy from mutations, or predictive methods like DbD2 (102), which predicts mutations to introduce disulfide bonds that potentially have stabilizing effects on the protein for given conditions. Potential mutations identified via heuristic methods are then commonly evaluated as a narrow combinatorial library. Although not strictly necessary, to reduce cost and labor for the in vitro experiments, the mutated proteins are often computationally evaluated for stability to 
further narrow down viable mutations. Because of their heuristic nature, it is always necessary to be able to introduce the mutations in vitro and evaluate them experimentally under the target conditions to confirm the mutated protein is improved.

Good, directed, and rational mutations will take into consideration the final target conditions. However, further tuning of the reaction environment may yield better performance while still remaining within viable conditions. Response Surface Methodology (RSM), within the field of optimization theory, provides a mathematical model to predict fitness as a function of controllable variables (103). Experimental frameworks used in RSM are generally tractable for a small number of independent variables. Work by Tripathi and Mishra (104) determined optimal conditions for $\mathrm{AFB}_{1}$ detoxification in red chili powder using three independent variables, requiring 20 experiments to solve a second order design. In summary, classical optimization of enzyme performance occurs in two stages. First, the enzyme is mutated to introduce desirable interactions with its substrate, and second, the mutated enzyme undergoes reaction condition optimization.

\section{Future Outlook}

\subsection{Advancements in tools that enable a mechanistic understanding}

Both the use of machine learning and automated, high-throughput optimization laboratory experiments are becoming increasingly prevalent for biological domains, enzyme engineering in particular. Enzyme engineering may become a useful tool for the optimization of known degrading enzymes, especially when only sequences, rather than solved crystallographic structures, are known (105). Models for directed evolution can be experimentally realized in parallel and incrementally updated, moving towards an optimal sequence. To obtain sequences, Top-Down Mass Spectroscopy (TD-MS) is one step in the direction of MS (106). Entire proteins are ionized and characterized, rather than the digested peptide fragments used in bottom-up (BUMS) approaches (107). TD-MS still faces difficulties in resolution power for proteins $>70 \mathrm{kDa}$, as well as stricter requirements for purity and concentration of the protein solution (108).

Methodologies that are not sensitive to sample noise are favorable for high-throughput screening and optimization, as they require less specialization and time spent in purification. A number of new techniques are emerging using the base concept of an electromagnetic fingerprint of the protein, either denatured or whole, which may overcome some of the difficulties seen in large mass resolution in TD-MS (109). 


\subsection{Machine learning and probabilistic models for computational biology}

Exciting new methods adjacent to mycotoxin degradation have emerged in the past few years, paving the way for efficient tools for understanding, identifying, and improving mycotoxin degradation. As described in Section 2, computational screening for mycotoxin degraders will encompass genomic, proteomic, and drug screening methodologies, thus relevant advances in these areas are pertinent.

There are two codependent tasks to consider in developing a degrader: the discovery of an enzymatic process that degrades the toxin, and an understanding of the process in order to leverage it for efficient degradation at scale. Finding the enzymatic process is still largely done experimentally, and its computational equivalent could accelerate and inform such efforts, while containing costs. Towards the understanding of enzymatic mechanisms, advancements in quantum mechanics (QM) and molecular mechanics (MM, atomistic) studies will be vital for characterizing reaction mechanisms and exploring the chemical space available via mutations. Additionally, crystallographic structures can be slow and expensive to solve; therefore, recent advances in protein 3D structure prediction will be instrumental to develop high-throughput pipelines.

Molecular mechanics provides an atomistic view of a system, and is often employed when the simulation is done at the atomic level. It is often used for molecular dynamics (MD) simulations, where a small system is studied using Newtonian physics, often at nanosecond to microsecond timescales. For some protein systems, this timescale is sufficient to study the relevant mechanisms; however, for larger proteins, or proteins involving large conformational shifts, extensive computation may be needed. For these systems, a coarse-grained approach is taken where moieties in the system are combined to reduce the total atom count, reducing the computational cost $(110,111)$. Some examples are coarse-grained water models, as well as proteins where the side chains are often reduced to a single pseudo-atom. Coarse-grained models pose significant issues in faithfully reproducing the potential energy surface of the system, and much research is focused in this area (111).

Atomistic systems allow non-electronic insight into the interaction between the protein and the toxin, without the prohibitive overhead of QM calculations. They are often sufficient to determine if the toxin will sterically fit in the binding pocket, and may also help to determine pose, electrostatic favorability of the binding, and conformational changes of the protein-ligand complex $(112,113)$. Unlike the more common use for MM in evaluating non-covalent inhibitors, some difficulty emerges in the inherent covalent nature of detoxification, which cannot be captured by an atomistic view (114). This may preclude some energetic effects brought about by the changes in electronic structure and one must be wary of what is exactly being measured by each level of theory. This may be partially solved by using QM/MM methods, where part of the system is partitioned into a QM region, and the rest remains in MM views (115). The QM region then can model electronic changes, and the rest can remain in lower cost MM regions. The QM 
region may not be too large, however, which often precludes metalloenzymes like laccases, which require large, complex QM regions. Additionally, the QM region adds additional computational cost and cannot be well-integrated into microsecond timescale calculations.

At a relatively high computational cost, QM calculations provide a detailed and comprehensive view of the electronic state of the system. They can provide information about covalent and electronic changes, often necessary for detoxification studies. An example of this is calculating a Fukui function of a molecule, which describes the change in a frontier orbital as the molecule undergoes a redox reaction. Fukui functions have been used to identify the location of redox in an AF-laccase system (52). QM may also be used to study electron transfer in the protein. As a tool for microbiologists, however, it remains prohibitively expensive both in computational cost and learning curve, and is often used for fine-grained mechanistic studies in collaboration with a QM expert.

\section{Acknowledgements}

This work was supported by Boston College through a startup fund as well as an Ignite grant.

\section{References}

1. Alshannaq A, Yu JH. 2017. Occurrence, toxicity, and analysis of major mycotoxins in food. Int J Environ Res Public Health. MDPI AG.

2. Marin S, Ramos AJ, Cano-Sancho G, Sanchis V. 2013. Mycotoxins: Occurrence, toxicology, and exposure assessment. Food Chem Toxicol. Food Chem Toxicol.

3. Colović R, Puvača N, Cheli F, Avantaggiato G, Greco D, Đuragić O, Kos J, Pinotti L. 2019. Decontamination of mycotoxin-contaminated feedstuffs and compound feed. Toxins (Basel). MDPI AG.

4. Kebede H, Liu X, Jin J, Xing F. 2020. Current status of major mycotoxins contamination in food and feed in Africa. Food Control. Elsevier Ltd.

5. Edite Bezerra da Rocha M, Freire F da CO, Erlan Feitosa Maia F, Izabel Florindo Guedes M, Rondina D. 2014. Mycotoxins and their effects on human and animal health. Food Control. Elsevier.

6. Bennett JW, Klich M. 2003. Mycotoxins. Clin Microbiol Rev 16:497-516.

7. Mahato DK, Lee KE, Kamle M, Devi S, Dewangan KN, Kumar P, Kang SG. 2019. Aflatoxins in Food and Feed: An Overview on Prevalence, Detection and Control Strategies. Front Microbiol. Frontiers Media S.A.

8. Liew WPP, Mohd-Redzwan S. 2018. Mycotoxin: Its impact on gut health and microbiota. Front Cell Infect Microbiol. Frontiers Media S.A.

9. Bui-Klimke TR, Wu F. 2015. Ochratoxin A and Human Health Risk: A Review of the Evidence. Crit Rev Food Sci Nutr 55:1860-1869.

10. Hueza IM, Raspantini PCF, Raspantini LER, Latorre AO, Górniak SL. 2014. Zearalenone, 
an estrogenic mycotoxin, is an immunotoxic compound. Toxins (Basel) 6:1080-1095.

11. Sydenham EW, Shephard GS, Thiel PG, Marasas WFO, Stockenström S. 1991.

Fumonisin Contamination of Commercial Corn-Based Human Foodstuffs. J Agric Food Chem 39:2014-2018.

12. Sobrova P, Adam V, Vasatkova A, Beklova M, Zeman L, Kizek R. 2010. Deoxynivalenol and its toxicity. Interdiscip Toxicol. Slovak Toxicology Society.

13. Pal S, Singh N, Ansari KM. 2017. Toxicological effects of patulin mycotoxin on the mammalian system: An overview. Toxicol Res (Camb). Royal Society of Chemistry.

14. Bhatnagar D, Payne G, Cleveland TE, Robens J. 2003. MYCOTOXINS: CURRENT ISSUES IN U.S.A. USDA ARS.

15. Pankaj SK, Shi H, Keener KM. 2018. A review of novel physical and chemical decontamination technologies for aflatoxin in food. Trends Food Sci Technol. Elsevier Ltd.

16. Karlovsky P, Suman M, Berthiller F, De Meester J, Eisenbrand G, Perrin I, Oswald IP, Speijers G, Chiodini A, Recker T, Dussort P. 2016. Impact of food processing and detoxification treatments on mycotoxin contamination. Mycotoxin Res. Springer Verlag.

17. Wu Q, Jezkova A, Yuan Z, Pavlikova L, Dohnal V, Kuca K. 2009. Biological degradation of aflatoxins. Drug Metab Rev 41:1-7.

18. Singh R, Singh P, Sharma R. 2014. Microorganism as a tool of bioremediation technology for cleaning environment: A reviewProceedings of the International Academy of Ecology and Environmental Sciences.

19. Grenier B, Loureiro-Bracarense A-P, Leslie JF, Oswald IP. 2014. Physical and Chemical Methods for Mycotoxin Decontamination in Maize, p. 116-129. In Mycotoxin Reduction in Grain Chains. John Wiley \& Sons, Ltd, Chichester, UK.

20. Abatenh E, Gizaw B, Tsegaye Z, Wassie M. 2017. The Role of Microorganisms in Bioremediation- A Review. Open J Environ Biol 2:038-046.

21. Agriopoulou S, Stamatelopoulou E, Varzakas T. 2020. Advances in occurrence, importance, and mycotoxin control strategies: Prevention and detoxification in foods. Foods. MDPI Multidisciplinary Digital Publishing Institute.

22. Kabak B, Dobson ADW, Var I. 2006. Strategies to Prevent Mycotoxin Contamination of Food and Animal Feed: A Review. Crit Rev Food Sci Nutr 46:593-619.

23. Perczak A, Goliński P, Bryła M, Waśkiewicz A. 2018. The efficiency of lactic acid bacteria against pathogenic fungi and mycotoxins. Arh Hig Rada Toksikol. Institute for Medical Research and Occupational Health.

24. Sadiq FA, Yan B, Tian F, Zhao J, Zhang H, Chen W. 2019. Lactic Acid Bacteria as Antifungal and Anti-Mycotoxigenic Agents: A Comprehensive Review. Compr Rev Food Sci Food Saf 18:1403-1436.

25. Muhialdin BJ, Saari N, Meor Hussin AS. 2020. Review on the Biological Detoxification of Mycotoxins Using Lactic Acid Bacteria to Enhance the Sustainability of Foods Supply. Molecules. NLM (Medline). 
26. de Melo Nazareth T, Luz C, Torrijos R, Quiles JM, Luciano FB, Mañes J, Meca G. 2019. Potential application of lactic acid bacteria to reduce aflatoxin B1 and fumonisin B1 occurrence on corn kernels and corn ears. Toxins (Basel) 12.

27. Vega MF, Dieguez SN, Riccio B, Aranguren S, Giordano A, Denzoin L, Soraci AL, Tapia MO, Ross R, Apás A, González SN. 2017. Zearalenone adsorption capacity of lactic acid bacteria isolated from pigs. Brazilian J Microbiol 48:715-723.

28. Loi M, Fanelli F, Liuzzi VC, Logrieco AF, Mulè G. 2017. Mycotoxin biotransformation by native and commercial enzymes: Present and future perspectives. Toxins (Basel). MDPI AG.

29. Alberts JF, Gelderblom WCA, Botha A, van Zyl WH. 2009. Degradation of aflatoxin B1 by fungal laccase enzymes. Int J Food Microbiol 135:47-52.

30. Tran VN, Viktorová J, Ruml T. 2020. Mycotoxins: Biotransformation and Bioavailability Assessment Using Caco-2 Cell Monolayer. Toxins .

31. Haskard CA, El-Nezami HS, Kankaanpää PE, Salminen S, Ahokas JT. 2001. Surface Binding of Aflatoxin B1 by Lactic Acid Bacteria. Appl Environ Microbiol 67:3086-3091.

32. Kim S, Lee H, Lee S, Lee J, Ha J, Choi Y, Yoon Y, Choi KH. 2017. Invited review: Microbe-mediated aflatoxin decontamination of dairy products and feeds. J Dairy Sci 100:871-880.

33. Kabak B, Var I. 2008. Factors affecting the removal of aflatoxin M1 from food model by Lactobacillus and Bifidobacterium strains. J Environ Sci Heal - Part B Pestic Food Contam Agric Wastes 43:617-624.

34. Piotrowska M. 2014. The adsorption of ochratoxin a by lactobacillus species. Toxins (Basel) 6:2826-2839.

35. Jard G, Liboz T, Mathieu F, Guyonvarc'H A, Lebrihi A. 2009. Adsorption of zearalenone by Aspergillus japonicus conidia: New trends for biological decontamination in animal feed. World Mycotoxin J 2:391-397.

36. Sangare L, Zhao Y, Folly YM inni. E, Chang J, Li J, Selvaraj JN ima., Xing F, Zhou L, Wang Y, Liu Y. 2014. Aflatoxin B 1 degradation by a Pseudomonas strain. Toxins (Basel) 6:3028-3040.

37. Alberts J, Engelbrecht Y, Steyn P, Holzapfel W, Zyl, Van W. 2006. Biological degradation of aflatoxin B1 by Rhodococcus erythropolis cultures. Int J Food Microbiol 109:121-126.

38. Guan S, Ji C, Zhou T, Li J, Ma Q, Niu T. 2008. Aflatoxin B1 Degradation by Stenotrophomonas Maltophilia and Other Microbes Selected Using Coumarin Medium. Int J Mol Sci 9:1489-1503.

39. Guan S, Zhao L, Ma Q, Zhou T, Wang N, Hu X, Ji C. 2010. In Vitro Efficacy of Myxococcus fulvus ANSM068 to Biotransform Aflatoxin B1. Int J Mol Sci 11:40634079.

40. Ji C, Fan Y, Zhao L. 2016. Review on biological degradation of mycotoxins. Anim Nutr. KeAi Communications Co. 
41. Sato I, Ito M, Ishizaka M, Ikunaga Y, Sato Y, Yoshida S, Koitabashi M, Tsushima S. 2012. Thirteen novel deoxynivalenol-degrading bacteria are classified within two genera with distinct degradation mechanisms. FEMS Microbiol Lett 327:110-117.

42. Adebo OA, Njobeh PB, Gbashi S, Nwinyi OC, Mavumengwana V. 2017. Review on microbial degradation of aflatoxins. Crit Rev Food Sci Nutr 57:3208-3217.

43. Lyagin I, Efremenko E. 2019. Enzymes for detoxification of various mycotoxins: Origins and mechanisms of catalytic action. Molecules. MDPI AG.

44. Zhu R, Feussner K, Wu T, Yan F, Karlovsky P, Zheng X. 2015. Detoxification of mycotoxin patulin by the yeast Rhodosporidium paludigenum. Food Chem 179:1-5.

45. Murugesan GR, Ledoux DR, Naehrer K, Berthiller F, Applegate TJ, Grenier B, Phillips TD, Schatzmayr G. 2015. Prevalence and effects of mycotoxins on poultry health and performance, and recent development in mycotoxin counteracting strategies, p. 12981315. In Poultry Science. Oxford University Press.

46. Heinl S, Hartinger D, Thamhesl M, Vekiru E, Krska R, Schatzmayr G, Moll WD, Grabherr R. 2010. Degradation of fumonisin B1 by the consecutive action of two bacterial enzymes. J Biotechnol 145:120-129.

47. Protein BLAST: search protein databases using a protein query.

48. Afsharmanesh H, Perez-Garcia A, Zeriouh H, Ahmadzadeh M, Romero D. 2018. Aflatoxin degradation by Bacillus subtilis UTB1 is based on production of an oxidoreductase involved in bacilysin biosynthesis. Food Control 94:48-55.

49. Dellafiora L, Galaverna G, Reverberi M, Dall'Asta C. 2017. Degradation of Aflatoxins by Means of Laccases from Trametes versicolor: An In Silico Insight. Toxins (Basel) 9:17.

50. Li Z, Wang Y, Liu Z, Jin S, Pan K, Liu H, Liu T, Li X, Zhang C, Luo X, Song Y, Zhao J, Zhang T. 2021. Biological detoxification of fumonisin by a novel carboxylesterase from Sphingomonadales bacterium and its biochemical characterization. Int J Biol Macromol 169:18-27.

51. Risa A, Divinyi DM, Baka E, Krifaton C. 2017. Aflatoxin B1 detoxification by cellfree extracts of Rhodococcus strains. Acta Microbiol Immunol Hung 64:423-438.

52. Zaccaria M, Dawson W, Kish DR, Reverberi M, Bonaccorsi Di Patti MC, Domin M, Cristiglio V, Dellafiora L, Gabel F, Nakajima T, Genovese L, Momeni B. 2021. Mechanistic Insight from Full Quantum Mechanical Modeling: Laccase as a Detoxifier of Aflatoxins. bioRxiv 2020.12.31.424992.

53. Humpf HU, Schmelz EM, Meredith FI, Vesper H, Vales TR, Wang E, Menaldino DS, Liotta DC, Merrill AH. 1998. Acylation of naturally occurring and synthetic 1deoxysphinganines by ceramide synthase: Formation of N-palmitoyl-aminopentol produces a toxic metabolite of hydrolyzed fumonisin, AP1, and a new category of ceramide synthase inhibitor. J Biol Chem 273:19060-19064.

54. Carere J, Hassan YI, Lepp D, Zhou T. 2018. The enzymatic detoxification of the mycotoxin deoxynivalenol: identification of DepA from the DON epimerization pathway. Microb Biotechnol 11:1106-1111. 
55. Carere J, Hassan YI, Lepp D, Zhou T. 2018. The identification of DepB: An enzyme responsible for the final detoxification step in the deoxynivalenol epimerization pathway in devosia mutans 17-2-E-8. Front Microbiol 9.

56. Altalhi AD, El-Deeb B. 2007. Localization of Zearalenone Detoxification Gene(s) in pZEA-1 Plasmid of Pseudomonas putida sp. Strain ZEA-1 and Expressed in Escherichia coli. Malaysian Society for Microbiology.

57. Hahn I, Kunz-Vekiru E, Twarużek M, Grajewski J, Krska R, Berthiller F. 2015. Aerobic and anaerobic in vitro testing of feed additives claiming to detoxify deoxynivalenol and zearalenone. Food Addit Contam - Part A Chem Anal Control Expo Risk Assess 32:922933.

58. Catteuw A, Broekaert N, De Baere S, Lauwers M, Gasthuys E, Huybrechts B, Callebaut A, Ivanova L, Uhlig S, De Boevre M, De Saeger S, Gehring R, Devreese M, Croubels S. 2019. Insights into in Vivo Absolute Oral Bioavailability, Biotransformation, and Toxicokinetics of Zearalenone, $\alpha$-Zearalenol, $\beta$-Zearalenol, Zearalenone-14-glucoside, and Zearalenone-14-sulfate in Pigs. J Agric Food Chem 67:3448-3458.

59. Wang Y, Zhao C, Zhang D, Zhao M, Peng M, Guo P, Cui Z. 2020. Microbial Degradation of Zearalenone by a Novel Microbial Consortium, NZDC-6, and Its Application on Contaminated Corncob by Semisolid Fermentation. J Agric Food Chem 68:1634-1644.

60. Bervoets I, Charlier D. 2019. Diversity, versatility and complexity of bacterial gene regulation mechanisms: Opportunities and drawbacks for applications in synthetic biology. FEMS Microbiol Rev. Oxford University Press.

61. Haugen SP, Ross W, Gourse RL. 2008. Advances in bacterial promoter recognition and its control by factors that do not bind DNA. Nat Rev Microbiol. Nat Rev Microbiol.

62. Maffei B, Francetic O, Subtil A. 2017. Tracking proteins secreted by bacteria: What's in the toolbox? Front Cell Infect Microbiol 7.

63. Klucar L, Stano M, Hajduk M. 2009. PhiSITE: Database of gene regulation in bacteriophages. Nucleic Acids Res 38.

64. Stano M, Klucar L. 2011. PhiGENOME: An integrative navigation throughout bacteriophage genomes. Genomics 98:376-380.

65. Coppens L, Lavigne R. 2020. SAPPHIRE: A neural network based classifier for $\sigma 70$ promoter prediction in Pseudomonas. BMC Bioinformatics 21.

66. Eckweiler D, Dudek CA, Hartlich J, Brötje D, Jahn D. 2018. PRODORIC2: The bacterial gene regulation database in 2018. Nucleic Acids Res 46:D320-D326.

67. de Avila e Silva S, Echeverrigaray S, Gerhardt GJL. 2011. BacPP: Bacterial promoter prediction-A tool for accurate sigma-factor specific assignment in enterobacteria. $\mathrm{J}$ Theor Biol 287:92-99.

68. Umarov RK, Solovyev V V. 2017. Recognition of prokaryotic and eukaryotic promoters using convolutional deep learning neural networks. PLoS One 12.

69. Osbourn AE, Field B. 2009. Operons. Cell Mol Life Sci. Cell Mol Life Sci.

70. Okuda S, Yoshizawa AC. 2011. ODB: A database for operon organizations, 2011 update. 
Nucleic Acids Res 39:D552.

71. ODB4 - Operon Database.

72. Chetal K, Janga SC. 2015. OperomeDB: A Database of Condition-Specific Transcription Units in Prokaryotic Genomes. Biomed Res Int 2015.

73. Multi Gene Operon Prediction for Bacterial Genomes Database.

74. Assaf R, Xia F, Stevens R. 2021. Detecting operons in bacterial genomes via visual representation learning. Sci Rep 11:2124.

75. Taboada B, Estrada K, Ciria R, Merino E. 2018. Operon-mapper: A web server for precise operon identification in bacterial and archaeal genomes. Bioinformatics 34:4118-4120.

76. Operon Mapper.

77. Seemann T. 2014. Prokka: Rapid prokaryotic genome annotation. Bioinformatics 30:2068-2069.

78. Eddy SR. 2011. Accelerated profile HMM searches. PLoS Comput Biol 7.

79. Jensen LJ, Kuhn M, Stark M, Chaffron S, Creevey C, Muller J, Doerks T, Julien P, Roth A, Simonovic M, Bork P, von Mering C. 2009. STRING 8 - A global view on proteins and their functional interactions in 630 organisms. Nucleic Acids Res 37.

80. Taboada B, Verde C, Merino E. 2010. High accuracy operon prediction method based on STRING database scores. Nucleic Acids Res 38.

81. Buchfink B, Xie C, Huson DH. 2014. Fast and sensitive protein alignment using DIAMOND. Nat Methods. Nature Publishing Group.

82. Zaidi SSA, Zhang X. 2017. Computational operon prediction in whole-genomes and metagenomes. Brief Funct Genomics 16:181-193.

83. Owji H, Nezafat N, Negahdaripour M, Hajiebrahimi A, Ghasemi Y. 2018. A comprehensive review of signal peptides: Structure, roles, and applications. Eur J Cell Biol. Elsevier GmbH.

84. Almagro Armenteros JJ, Tsirigos KD, Sønderby CK, Petersen TN, Winther O, Brunak S, von Heijne G, Nielsen H. 2019. SignalP 5.0 improves signal peptide predictions using deep neural networks. Nat Biotechnol 37:420-423.

85. Yu NY, Wagner JR, Laird MR, Melli G, Rey S, Lo R, Dao P, Cenk Sahinalp S, Ester M, Foster LJ, Brinkman FSL. 2010. PSORTb 3.0: Improved protein subcellular localization prediction with refined localization subcategories and predictive capabilities for all prokaryotes. Bioinformatics 26:1608-1615.

86. Bagos PG, Nikolaou EP, Liakopoulos TD, Tsirigos KD. 2010. Combined prediction of Tat and Sec signal peptides with hidden Markov models. Bioinformatics 26:2811-2817.

87. Bendtsen JD, Nielsen H, Widdick D, Palmer T, Brunak S. 2005. Prediction of twinarginine signal peptides. BMC Bioinformatics 6.

88. Nielsen H, Engelbrecht J, Brunak S, Von Heijne G. 1997. Identification of prokaryotic and eukaryotic signal peptides and prediction of their cleavage sites. Protein Eng 10:1-6. 
89. Krogh AS, Nielsen H. 1998. Prediction of signal peptides and signal anchors by a hidden Markov model., p. 122-130. In Proc Int Conf Intell Syst Mol Biol. AAAI Press.

90. Bendtsen JD, Nielsen H, Von Heijne G, Brunak S. 2004. Improved prediction of signal peptides: SignalP 3.0. J Mol Biol 340:783-795.

91. Petersen TN, Brunak S, Von Heijne G, Nielsen H. 2011. SignalP 4.0: Discriminating signal peptides from transmembrane regions. Nat Methods. Nat Methods.

92. Farinas ET, Bulter T, Arnold FH. 2001. Directed enzyme evolution. Curr Opin Biotechnol. Elsevier Ltd.

93. Lutz S, Patrick WM. 2004. Novel methods for directed evolution of enzymes: Quality, not quantity. Curr Opin Biotechnol. Curr Opin Biotechnol.

94. Goldsmith M, Tawfik DS. 2012. Directed enzyme evolution: Beyond the low-hanging fruit. Curr Opin Struct Biol. Elsevier Current Trends.

95. Morley KL, Kazlauskas RJ. 2005. Improving enzyme properties: When are closer mutations better? Trends Biotechnol 23:231-237.

96. Chica RA, Doucet N, Pelletier JN. 2005. Semi-rational approaches to engineering enzyme activity: Combining the benefits of directed evolution and rational design. Curr Opin Biotechnol. Curr Opin Biotechnol.

97. Siedhoff NE, Schwaneberg U, Davari MD. 2020. Machine learning-assisted enzyme engineering, p. 281-315. In Methods in Enzymology. Academic Press Inc.

98. Berland M, Offmann B, André I, Remaud-Siméon M, Charton P, Arnold F. 2014. Awebbased tool for rational screening of mutants libraries using ProSAR, p. 375-381. In Protein Engineering, Design and Selection. Oxford University Press.

99. Fox RJ, Davis SC, Mundorff EC, Newman LM, Gavrilovic V, Ma SK, Chung LM, Ching C, Tam S, Muley S, Grate J, Gruber J, Whitman JC, Sheldon RA, Huisman GW. 2007. Improving catalytic function by ProSAR-driven enzyme evolution. Nat Biotechnol 25:338-344.

100. Alley EC, Khimulya G, Biswas S, AlQuraishi M, Church GM. 2019. Unified rational protein engineering with sequence-based deep representation learning. Nat Methods 16:1315-1322.

101. Schymkowitz J, Borg J, Stricher F, Nys R, Rousseau F, Serrano L. 2005. The FoldX web server: An online force field. Nucleic Acids Res 33:W382.

102. Craig DB, Dombkowski AA. 2013. Disulfide by Design 2.0: A web-based tool for disulfide engineering in proteins. BMC Bioinformatics 14:346.

103. Khuri AI, Mukhopadhyay S. 2010. Response surface methodology. Wiley Interdiscip Rev Comput Stat 2:128-149.

104. Tripathi S, Mishra HN. 2011. Modeling and Optimization of Enzymatic Degradation of Aflatoxin B 1 (AFB 1) in Red Chili Powder Using Response Surface Methodology. Food Bioprocess Technol 4:770-780.

105. Chowdhury R, Maranas CD. 2020. From directed evolution to computational enzyme engineering-A review. AIChE J 66:e16847. 
106. Zhou M, Lantz C, Brown KA, Ge Y, Paša-Tolić L, Loo JA, Lermyte F. 2020. Higherorder structural characterisation of native proteins and complexes by top-down mass spectrometry. Chem Sci. Royal Society of Chemistry.

107. Kelleher NL, Lin HY, Valaskovic GA, Aaserud DJ, Fridriksson EK, McLafferty FW. 1999. Top down versus bottom up protein characterization by tandem high- resolution mass spectrometry. J Am Chem Soc 121:806-812.

108. Cupp-Sutton KA, Wu S. 2020. High-throughput quantitative top-down proteomics. Mol Omi. Royal Society of Chemistry.

109. Timp W, Timp G. 2020. Beyond mass spectrometry, the next step in proteomics. Sci Adv 6:eaax 8978.

110. Souza PCT, Alessandri R, Barnoud J, Thallmair S, Faustino I, Grünewald F, Patmanidis I, Abdizadeh H, Bruininks BMH, Wassenaar TA, Kroon PC, Melcr J, Nieto V, Corradi V, Khan HM, Domański J, Javanainen M, Martinez-Seara H, Reuter N, Best RB, Vattulainen I, Monticelli L, Periole X, Tieleman DP, de Vries AH, Marrink SJ. 2021. Martini 3: a general purpose force field for coarse-grained molecular dynamics. Nat Methods 18:382388.

111. Wang J, Chmiela S, Müller KR, Noé F, Clementi C. 2020. Ensemble learning of coarsegrained molecular dynamics force fields with a kernel approach. arXiv. arXiv.

112. Guterres H, Im W. 2020. Improving Protein-Ligand Docking Results with HighThroughput Molecular Dynamics Simulations. J Chem Inf Model 60:2189-2198.

113. Peterson L. 2020. In Silico Molecular Dynamics Docking of Drugs to the Inhibitory Active Site of SARS-CoV-2 Protease and Their Predicted Toxicology and ADME. SSRN Electron J https://doi.org/10.2139/ssrn.3580951.

114. Aljoundi A, Bjij I, El Rashedy A, Soliman MES. 2020. Covalent Versus Non-covalent Enzyme Inhibition: Which Route Should We Take? A Justification of the Good and Bad from Molecular Modelling Perspective. Protein J. Springer.

115. Yu T, Guo H. 2020. Understanding Enzyme Catalysis Mechanism Using QM/MM Simulation Methods, p. 121-137. In ACS Symposium Series. American Chemical Society. 